\title{
A Recommendation for New Diction Rhymes to be used in Turkish Diction Education Studies
}

\section{Ismail COBAN}

Artvin Çoruh University, Faculty of Education, Turkish Language Teaching Department Turkey. Email:smlcoban@gmail.com Tel: +905362844893

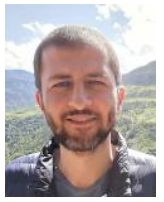

\begin{abstract}
Acquisition of the ability to speak correctly and effectively is one of the main purposes of elocution. One of the conditions required to achieve this is to have a good diction. Although the students speak standard Turkish language at schools, they have poor diction due to being under the influence of local dialects in the family and outside the school. This affects the process of making the students acquire the ability of speaking, one of the main objectives of Turkish teaching. The aim of the study addressed with case study method is to determine how the diction rhymes should be to be prepared by the prospective Turkish teachers in order to correct and improve the diction of the students. The study group constituted 46 first-grade prospective Turkish teachers studying in the Department of Turkish Language Education, in Ataturk University between 2017-2018 academic year. Prospective Turkish teachers were asked to create a diction rhyme for 10 sounds "b, p, d, t, g, k, e, o, u, ü". 434 diction rhymes were obtained as a result of the research. Rhymes were examined in terms of length, including the number of the target sound, entertainment, creativity, and availability in diction training. As a result of the examination, 149 of the recommended rhymes were determined to have the capability to be used in diction training.
\end{abstract}

Keywords: Diction, Sentence, Rhyme, Availability, Length, Creativity, Entertaining, Prospective Turkish teachers.

Citation | Ismail COBAN (2020). A Recommendation for New Diction Rhymes to be Used in Turkish Diction Education Studies. Asian Journal of Education and Training, 6(3): 347-355.

History:

Received: 6 March 2020

Revised: 10 April 2020

Accepted: 14 May 2020

Published: 8 June 2020

Licensed: This work is licensed under a Creative Commons

Attribution 3.0 License $(\mathrm{cc})$ E

Publisher: Asian Online Journal Publishing Group
Funding: This study received no specific financial support.

Competing Interests: The author declares that there are no conflicts of interests regarding the publication of this paper.

Transparency: The author confirms that the manuscript is an honest, Transparency: The author confirms that the manuscript is an honest,
accurate, and transparent account of the study was reported; that no vital features of the study have been omitted; and that any discrepancies from the features of the study have been omit
study as planned have been explained.

Ethical: This study follows all ethical practices during writing.

\section{Contents}

1. Introduction

4. Conclusion and Recommendations

References. 


\section{Contribution of this paper to the literature}

This study contributes to existing literature by recommending new diction rhymes to be used in Turkish diction education studies and also determines prospective Turkish Teachers' skills of creating diction rhymes.

\section{Introduction}

Considering the contribution of socialization to success, it is significant to obtain effective and efficient use of verbal communication skills. "Language acquisition begins as a result of the interaction of the child with her family and close circle before school" (Alyılmaz, 2010). It is one of the main purposes to make the students gain verbal communication skills in a world where the benefits of communication are increasing.

"Anything we do with other people must involve communication" (Hartley, 2010). The major provider / tool of the communication is speech. Speech is a psychophysical process formed by sound-light-waves arising from the movement of one's muscles in order to influence an individual/individuals (Taşer, 2009). The individual must have some characteristics in order to be successful in this process. One of them is to have a good diction.

Diction is the art of using the sound correctly to express feelings and thoughts in accordance with the style of speech while speaking. For this purpose, it is the application of the harmony of the sound, the pronunciation, the length of the word syllables in proper positions. The articulation points of the sounds, intonation, emphasis, liaison, stops and harmony of the rhythm with the words bring the success of diction. In addition, it is necessary to focus on the importance of body language supported by gestures appropriate to the word (Temizyürek, Erdem, \& Temizkan, 2013). A good diction is up to the ability to make sounds according to their articulation characteristics.

Articulation means that the sound path taking specific positions while making sounds. If these positions are performed correctly and in-position according to the structure of the language and the rules of speech, the sounds will be complete and correct and thus the speech will gain harmony. This is why articulation is considered the basis of diction (Gündüz \& Şimşek, 2014). An important issue in beautiful and effective speech is the perfect output of the sound. The perfect output of the sound requires a successful harmony between the output of the sound and the use of breath (Vural, 2007). Considering that language is a means of communication, pronunciation has priority in language learning, and learning pronunciation is of great importance for excellent verbal communication (Aydin \& Akyüz, 2017).

Environmental conditions, characteristics of individuals, customs and traditions sometimes cause the failure of pronunciation practices and not to be realized. "One of the possible reasons why some teachers do not prioritize pronunciations in their course curricula is that they think pronunciation is not a fundamental component of language teaching" (Cox, Henrichsen, Tanner, \& McMurry, 2019).

"Studies can be carried out to pronounce vowels and consonants primarily in order to pronounce sounds and words correctly and to improve speech training. Rhymes can be used for such purposes" (Gündüz \& Şimşek, 2014). Rhymes are phrases composed of alliterations to be used in articulation training. In rhyme training, it is necessary to get rid of the misunderstanding that they should be said very quickly. Rhymes must have simple or complex meanings or they should not have any meaning. Thus the semantic shift in the mind is prevented (Erdem, 2013). These rhymes, also known as fallacies, are particularly effective in children's language development due to the difficult utterances. When these rhymes formed by a combination of long and difficult words and letters mixed together according to their place of formation are tried to be said quickly or accurately. Funny situations can be experienced accordingly. Children who enjoy these funny situations will be willing to learn and use these rhymes (Dedeoğlu, 2009). Rhymes have an important place in pronunciations by the "tongue" in a certain position of the mouth increasing the movement function of the lower jaw, palate and tongue while the rhymes are said at various speeds (Toker, Sevinç, \& Önder, 2018).

In fallacies, there are traps that cause misunderstandings when the rhyme is read quickly and repeatedly, and these traps leave the speaker in a difficult position. The ineptitude of the trapped, or the strangeness of what the speaker says without even realizing it, amuses the ones listening. Some fallacies are in the form of poetry, while some are formed by the use of hard-to-say proverbs. Fallacies, which help children to speak beautifully and accurately, have the features of sleight of mouth (Yardımc1, 2004).

One of the most important practices that will improve the students' education about sound, emphasis and intonation is the repetition of rhymes. Rhymes to be used by the teachers after identifying the diction inadequacies of their students are essential materials for beautiful speaking. The teacher can make the students sing rhymes when the time and place is right. The practice of repeating a rhyme until the best one is said by the students can be performed. The ones succeeded in this practice can be rewarded. For these training, students may use rhymes either previously learned or to be heard in the class for the first time (Temizyürek, 2007). Rhymes are also used to the correct the diction disorders. Teaching the physical pronunciation of sound is important to get rid of the lisps. The contact point of the tongue should be shifted to the lower gingiva in order to get rid of the lisp pronunciation of the $\mathrm{s}$ and $\mathrm{z}$ sound that occurs when the tongue contacts between the teeth and pronunciation should be performed in the right position. Lisps should be tried to be eliminated by trying to pronounce rhymes related to these sounds and words (Erdem, 2013).

There are different uses according to the regions of a country where a language is spoken. These differences considered within the scope of local dialects cause individuals to make negative transfers from them while they speak the common language. A good verbal communication requires clear sounds, correct pronunciation of words and their transfer to the sentence level.

Correct pronunciation of sounds is successful with diction practices. One of the methods often used in diction improvement practice is the use of rhymes / exercise sentences. These sentences can be used in diction practice as well as in the training of correcting the individual's own diction. The length of these sentences should be well adjusted, involve the sufficient number of the sound to be improved, and be fun. According to Tanrikulu (2017) Turkish teachers find it "positive to include diction and rhymes" in the evaluation of the contents in EIN (Education Informatics Network). 
Çifci (2006) suggests that "teachers must be provided with adequate diction training in their undergraduate education so that they can give diction training to their students and become the right model". Er and Demir (2013) drew attention to the results of local dialect, inadequacy, fatigue, question-answer, boring expression and monotonous language use for a lively and fluent style among the negative views on the pattern and texture expressed by Turkish teachers at schools where the prospective Turkish teachers work as trainees.

Türk (2017) also highlighted this situation and stated that rhymes could be used in such cases. It is important for Turkish teachers to pronounce the words correctly. Pronunciation errors are seen as the inability to speak according to some experts. Inability to speak can be minimized by practices such as rhyme exercises, breathing exercises, training of speech muscles, drama, pantomime, theatre and oral reading. Performing exercises expressed to completely eliminate mispronunciations can be a beneficial process for the use of correct pronunciations (Türk, 2017).

Senyiğit and Okur (2019) state that rhymes can be used in pronunciation training for teaching Turkish to foreigners. Erdem, Şengül, Gün, and Büyükaslan (2015) also highlighted the use of a similar case for teaching Turkish to foreigners.

It has become possible to find rhymes in renewed educational and training settings. Especially the websites having educational content come to the forefront for this issue. "The rhymes given under the titles 'Diction Rhymes' which is the second category on the righthand side of the site and 'Twists-Fallacies' which can be found in the eighth category are intended to improve children's language skills and so that they can pronounce words correctly. These rhymes consisting of words that are difficult to say phonetically, outnumber the others. And there are registered 117 rhymes under the title 'Diction Rhymes' and 340 in the category 'Twists-Fallacies'. Considering the diction rhyme 'Bir berber bir berbere bre berber beri gel diye bar bar bağırmış. Bizde bize biz derler, sizde bize ne derler?' and twists-fallacies 'Kırk kırık küp, kırkının da kulpu kırık kara küp', it is possible to say that the rhyme texts in the site are composed largely of rhyme texts generally expressed in the oral tradition” (Büyükokutan, 2017).

The aim of education and training of Turkish language is to provide students with the ability to use verbal language skills effectively as well. This study was conducted to determine the skills of prospective Turkish teachers (PTT) to create diction rhymes to be used in order to improve the pronunciation when they have students having bad pronunciation in their professional lives. In the study, the answer to the question "What is the diction rhyme development skills of prospective Turkish teachers?" was sought. The answers for the following questions were also sought to answer the above mention question:

1. How is the length of diction rhymes prepared by prospective Turkish teachers?

2. How much does the diction rhymes prepared by the prospective Turkish teachers contain the sound intended to be developed?

3. Is the entertainment factor taken into account in the prepared rhymes?

4. What is the level of creativity of rhymes?

5. What is the availability of rhymes in diction improvement practice?

\section{Method}

Case study method from qualitative research methods was used in the study. Case study is "An in-depth depiction and analysis of a limited system" (Merriam, 2013). "It is the examination of a complex, special and interesting phenomenon and situation within their own circumstances” (Sönmez \& Alacapınar, 2014).

\subsection{Research Group}

During the determination of the research group of the study, easily accessible case sampling method, one of the intended sampling methods, was used. "This sampling method accelerates the study and provides practicality because the researcher selects a case that is close and easy to access with this method” (Yıldırım \& Şimşek, 2013). The research group constitutes 46 first-grade prospective Turkish teachers studying in the Department of Turkish Language Education, in Atatürk University between 2017-2018 academic year.

\subsection{Collection and Analysis of Data}

After 46 first-grade prospective Turkish teachers studying in Atatürk University, Department of Turkish Language Teaching were informed in the Verbal Lecture class about the features diction rhymes must have, they were asked to create diction rhymes for $b, p, d, t, g, k, e, o, u, u ̈$ sounds to determine what kind of sentences they can prepare to improve the poor diction of students when they become teachers. 434 diction rhymes were obtained due to rhymes that could not be created for some sounds. The data obtained were analyzed with the content analysis method. "(B), (P), (Ü)" was added to the rhyme formed by each prospective Turkish teacher with abbreviations such as $\mathrm{PTT}_{1}, \mathrm{PTT}_{2}, \mathrm{PTT}_{3}$, indicating which sound the rhyme was created for. When giving rhymes, an alphabetical order was preferred from being available to being unavailable.

Rhymes have been analyzed in terms of their length, how much they contain the sound to be developed, being entertaining and creative, and their availability. When evaluating how much the sentences contain the desired sound, 1-5 interval was determined to be less; 6-10 interval was determined to be medium; 11 and more were determined to be sufficient.

While analyzing the sentences, 1 Dr. and 1 Prof. working in the Department of Turkish Language were the score-givers. A consensus has been reached where different ideas were found. 


\section{Results}

Table-1. Length status of diction rhymes.

\begin{tabular}{l|l|l|l}
\hline & & $\mathbf{f}$ & \% \\
\hline \multirow{3}{*}{ Length } & Short & 152 & 35 \\
\cline { 2 - 4 } & Normal & 245 & 56,5 \\
\cline { 2 - 4 } & Long & 37 & 8,5 \\
\hline
\end{tabular}

When Table 1 is examined, it can be seen that $152(35 \%)$ of the suggested rhymes are short, $245(56.5 \%)$ are normal and $37(8.5 \%)$ are long. The suggested rhymes appear to consist mainly sentences of normal length.

\begin{tabular}{l|c|c|c}
\multicolumn{4}{|c}{ Table-2. Sound hosting status of diction rhymes. } \\
\hline & & $\mathbf{f}$ & $\mathbf{\%}$ \\
\hline \multirow{3}{*}{ Sound hosting } & Less & 98 & 22,6 \\
\cline { 2 - 4 } & Medium & 235 & 54,1 \\
\cline { 2 - 4 } & Sufficient & 101 & 23,3 \\
\hline
\end{tabular}

According to Table 2 analysis related how much place was given to the sound desired to be improved, it was determined that $98(22,6 \%)$ rhymes had less target sound, $235(54,1 \%)$ rhymes had medium and $101(23,3 \%)$ rhymes had sufficient level. It has been determined that the suggested rhymes had a positive impression in terms of giving the target sound a sufficient number of places.

\begin{tabular}{l|c|c|c}
\multicolumn{5}{c}{ Table-3. Entertaining status of diction rhymes. } & \% \\
\hline \multirow{3}{*}{ Entertaining } & & $\mathbf{f}$ & 33,2 \\
\cline { 2 - 4 } & Low & 144 & 39,6 \\
\cline { 2 - 4 } & Moderate & 172 & 27,2 \\
\hline
\end{tabular}

According to the information given in Table 3, it is shown that 144 (33.2\%) rhymes contain a low rate in terms of entertainment. It has been found that a moderate level of entertainment was in $172(39.6 \%)$ rhyme. It can be seen that $118(27.2 \%)$ sentences seemed to have the sufficiently entertaining qualification. It can be noted that the criteria for being entertaining in rhymes are adequately provided.

Table-4. Creativity status of diction rhymes.

\begin{tabular}{l|c|c|c}
\hline & & $\mathbf{f}$ & $\mathbf{\%}$ \\
\hline \multirow{3}{*}{ Creativity } & Insufficient & 161 & 37,1 \\
\cline { 2 - 4 } & Average & 152 & 35 \\
\cline { 2 - 4 } & Sufficient & 121 & 27,9 \\
\hline
\end{tabular}

When Table 4 is examined it was found that 161 (37.1\%) rhymes were insufficient in terms of creativity. Rhymes widely used and known by everyone were also evaluated under this title. 152 of the recommended rhymes $(35 \%)$ were found to be average in terms of creativity. 121 of the rhymes (27.9\%) have a sufficient level of creativity. It is noted that rhymes are found to be above-average in terms of creativity.

\begin{tabular}{l|c|c|c}
\multicolumn{4}{c}{ Table-5. Availability of diction rhymes. } \\
\hline & & $\mathbf{f}$ & \% \\
\hline \multirow{3}{*}{ Availability } & Unavailable & 155 & 35,7 \\
\cline { 2 - 4 } & Partly available & 130 & 30 \\
\cline { 2 - 4 } & Available & 149 & 34,3 \\
\hline
\end{tabular}

As can be seen in Table 5, 155 (35.7\%) of the recommended rhymes to be used in diction practices were found to be below the level of availability for diction practices. 130 of the rhymes (30\%) were found to be partially usable in diction exercises. 149 rhymes (34.3\%) were found to be suitable for diction exercises. The rhymes already used for diction exercises and suggested by the participants were also evaluated under this title. It was found that $64.3 \%$ of the recommended diction rhymes can be used in diction exercises.

It is possible to draw attention to the rhymes determined to be useful in diction practices from the suggested rhymes as follows:

Table-6. Available diction rhymes created by prospective Turkish teachers.

\begin{tabular}{|c|c|c|c|c|}
\hline Diction Rhymes & Length & $\begin{array}{l}\text { Sound } \\
\text { hosting }\end{array}$ & Entertaining & Creativity \\
\hline $\begin{array}{l}\text { A be ben sana beni bu kadar absürt beyle bir araya } \\
\text { getirme diye bir băg balıkla biraz da baklava vermedim } \\
\text { mi? } \mathrm{PTT}_{38}(\mathrm{~B})\end{array}$ & Long & Sufficient & Sufficiently & Sufficient \\
\hline $\begin{array}{l}\text { A be kuru dayı ne kuru sarı darı bu darı a be kuru dayı. } \\
\mathrm{PTT}_{38} \text { (D) }\end{array}$ & Normal & Less & Sufficiently & Insufficient \\
\hline $\begin{array}{l}\text { Al bu takatukaları takatukacıya götür. Takatukacı } \\
\text { takatukaları takatukalamazsa } \\
\text { takatukalatmadan getir. } \mathrm{PTT}_{14}(\mathrm{~T})\end{array}$ & Long & Sufficient & Sufficiently & Insufficient \\
\hline Al bu takatukaları takatukacıya götür. Takatukacı & Long & Sufficient & Sufficiently & Insufficient \\
\hline
\end{tabular}


takatukaları takatukalamazsa bana geri getir. $\mathrm{PTT}_{2}$

(T)

Al bu takatukaları takatukacıya götür. Takatukacı takatukaları takatukalamazsa geri bana getir. $\mathrm{PTT}_{23}$ (T)

Al bu takatukaları takatukacıya götür. Takatukacı takatukaları takatukalamazsa bana geri getir. PTT $_{39}$ (T)

Babamın babama babasindan kalan Bartın'daki evine babamla birlikte gittik. PTT $_{23}(\mathrm{~B})$

Bak bak bal gibi bol bol bal. $\mathrm{PTT}_{24}(\mathrm{~B})$

Bara gelen berber ile bardan gelen berber beraber pirelendi. $\mathrm{PTT}_{36}(\mathrm{~B})$

Beli belendi yedi baland yattı belendi kalktı balandı. $\mathrm{PTT}_{33}(\mathrm{~B})$

Ben bana baka baka biber biberi bala batıra batıra kandırmış. $\mathrm{PTT}_{4}(\mathrm{~B})$

Ben benden bene bütün benceleri benlerle bombalayn büblettim. $\mathrm{PTT}_{41}(\mathrm{~B})$

Ben beni bilirim ben beni, ben beni bilirim ben beni bilirim, ben beni bilmezsem patlaturlar ensemi. $\mathrm{PTT}_{21}$ (B)

Berber berbere "Bre berber barbar berber batıli berber" der. PTT $_{35}$ (B)

Bir berber bir berbere bre berber gel beraber bir berber dükkânı açalım demiş. $\mathrm{PTT}_{31}(\mathrm{~B})$

Bir berber bir berbere gel beraber Berberistan'da bir berber dükkânı açalım demiş. $\mathrm{PTT}_{45}(\mathrm{~B})$

Bir berber bir berbere gel beraber bir berberci dükkânı açalım demiş. $\mathrm{PTT}_{22}$ (B)

Bir berber bir berbere gel beraber bir berberci dükkânı açalım demiş. $\mathrm{PTT}_{39}(\mathrm{~B})$

Bir berber bir berbere gel bir berber dükkânı açalım

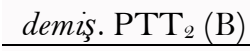

Bir berber bir berbere gel bir berber dükkânı açalım demiş. PTT $_{34}(\mathrm{~B})$

Bitli bact, bitli bakkal Bilal ile bitlenince nasıl bitli bitlerinden kurtulacaklarını aramaya başlardılar. $\mathrm{PTT}_{15}$ (B)

Bitli bebek büyük biberonla bitsiz sütü bitiledi. $\mathrm{PTT}_{37}$ (B)

Boş yere bırbır oluşturmayı bırak da bırbırlarını başka birbircıya sat. $\mathrm{PTT}_{42}(\mathrm{~B})$

Buradan Bursa'ya bașlayan bu tur Bursa'dan Burdur'u bulana dek son bulamadı. $\mathrm{PTT}_{7}(\mathrm{~B})$

Dal sarkar kartal kalkar, kartal kalkar dal sarkar.

$\mathrm{PTT}_{45}$ (D)

Daldan dala daldan dala dal kırıldı, daldan yere çakıldı.

$\mathrm{PTT}_{39}$ (D)

Daldan dala dallanan dalları budamaya, dal ile dallanan dalları görmeye dalından kopan çiçeğin dalından sallanan dalın, dalını dalından kesmeye gidiyormus. $\mathrm{PTT}_{5}$ (D)

Dededen denemeden de derin derelere düştü. $\mathrm{PTT}_{16}(\mathrm{D})$

Değirmene girdi köpek değirmenden çaldı kepek hem dayak yedi köpek hem kepek yedi köpek. $\mathrm{PTT}_{45}$ (D)

Deli Danyal deli deli bakarken birden delirmeye başladı. $\mathrm{PTT}_{14}(\mathrm{D})$

Delik deşik durdu duvar dedem tepindi dam dam. PTT $_{37}$ (D)

Demirci demirleri darbelerle dolayiversin demirletmeden de darbelesin. $\mathrm{PTT}_{35}(\mathrm{D})$

$\mathrm{D} \imath \mathrm{m}$ dım diyip dımdımlandırılıp $d_{\imath p} \quad d \imath p$ diyip dipdiplendirilmeden dimdik durdurulup don don dondurulmalı dondurmayz. PTT $_{41}$ (D)

Dondurma doldursak da mi dondursak dondurmasak da mı doldursak. $\mathrm{PTT}_{33}$ (D)

Döndü’nün dünyası düğünde döne döne döner dilimlemektir. $\mathrm{PTT}_{3}(\mathrm{D})$

Dura dura dul kalan Durmuş Dayı durduğu yerde durmaya doyamadan durmadan yürüyüp durakta durmuş. $\mathrm{PTT}_{6}$ (D)

Dün Derya ile Deniz dünyaya dalınca dedeleri Dündar, "Dünyaya dalmayın, dünyalık dünyada kalır" demiş.

\begin{tabular}{|c|c|c|c|}
\hline Long & Sufficient & Sufficiently & Insufficient \\
\hline Long & Sufficient & Sufficiently & Insufficient \\
\hline Normal & Medium & Moderate & Average \\
\hline Short & Medium & Sufficiently & Sufficient \\
\hline Normal & Medium & Moderate & Sufficient \\
\hline Normal & Less & Moderate & Sufficient \\
\hline Normal & Medium & Sufficiently & Sufficient \\
\hline Normal & Medium & Moderate & Average \\
\hline Normal & Sufficient & Sufficiently & Insufficient \\
\hline Normal & Sufficient & Moderate & Sufficient \\
\hline Normal & Sufficient & Moderate & Insufficient \\
\hline Normal & Sufficient & Moderate & Insufficient \\
\hline Normal & Sufficient & Low & Insufficient \\
\hline Normal & Sufficient & Moderate & Insufficient \\
\hline Normal & Medium & Moderate & Insufficient \\
\hline Normal & Medium & Moderate & Insufficient \\
\hline Normal & Medium & Sufficiently & Sufficient \\
\hline Normal & Medium & Moderate & Sufficient \\
\hline Normal & Medium & Moderate & Sufficient \\
\hline Normal & Medium & Moderate & Sufficient \\
\hline Normal & Medium & Sufficiently & Insufficient \\
\hline Normal & Sufficient & Sufficiently & Sufficient \\
\hline Long & Sufficient & Sufficiently & Sufficient \\
\hline Short & Medium & Moderate & Sufficient \\
\hline Normal & Medium & Sufficiently & Insufficient \\
\hline Normal & Medium & Sufficiently & Sufficient \\
\hline Normal & Medium & Sufficiently & Sufficient \\
\hline Normal & Medium & Moderate & Sufficient \\
\hline Long & Sufficient & Sufficiently & Sufficient \\
\hline Normal & Sufficient & Sufficiently & Sufficient \\
\hline Normal & Medium & Sufficiently & Sufficient \\
\hline Long & Sufficient & Sufficiently & Sufficient \\
\hline Long & Sufficient & Moderate & Average \\
\hline
\end{tabular}




\begin{tabular}{|c|c|c|c|c|}
\hline $\mathrm{PTT}_{13}(\mathrm{D})$ & & & & \\
\hline $\begin{array}{l}\text { Ebe gümeci ebe gümeleri ebe gümeleyenleri eliyle elletti. } \\
\mathrm{PTT}_{2}(\mathrm{E})\end{array}$ & Normal & Sufficient & Moderate & Sufficient \\
\hline $\begin{array}{l}\text { Edirneli ellitik Emin Emmi elmaların elli beş liraya } \\
\text { edindi. } \mathrm{PTT}_{31}(\mathrm{E})\end{array}$ & Normal & Medium & Sufficiently & Sufficient \\
\hline $\begin{array}{l}\text { El eder söz eder Erdem oğlan çok sever gelinin entarisini } \\
\text { el öper. } \mathrm{PTT}_{45}(\mathrm{E})\end{array}$ & Normal & Sufficient & Sufficiently & Sufficient \\
\hline El ele verilir, eşek yükü un elenir. $\mathrm{PTT}_{37}(\mathrm{E})$ & Short & Medium & Sufficiently & Sufficient \\
\hline El ele vermezsen benimle el ele verirsin el ile. $\mathrm{PTT}_{13}(\mathrm{E})$ & Normal & Sufficient & Moderate & Sufficient \\
\hline 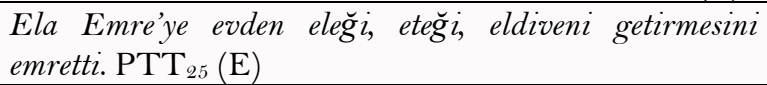 & Normal & Sufficient & Moderate & Sufficient \\
\hline $\begin{array}{l}\text { Elalem bir ev alip evlendi de biz daha bir ev alıp } \\
\text { evlenemedik. } \mathrm{PTT}_{15}(\mathrm{E})\end{array}$ & Normal & Sufficient & Moderate & Insufficient \\
\hline $\begin{array}{l}\text { Elalem ele gözlü Esma'nın ellerini es es eledi de esti. } \\
\mathrm{PTT}_{12}(\mathrm{E})\end{array}$ & Normal & Sufficient & Moderate & Average \\
\hline Elalem elinde elma, ey ehlin endamı Selma. $\mathrm{PTT}_{26}(\mathrm{E})$ & Normal & Medium & Sufficiently & Sufficient \\
\hline $\begin{array}{l}\text { Eledim eledim ununu, eyledim ettim senin sonunu. } \mathrm{PTT}_{27} \\
(\mathrm{E})\end{array}$ & Normal & Medium & Sufficiently & Sufficient \\
\hline $\begin{array}{l}\text { Elindeki elli liranın elli kuruşlarından elli tane elli kuruş } \\
\text { alıp ellere dağ } t . \mathrm{PTT}_{42}(\mathrm{E})\end{array}$ & Normal & Medium & Sufficiently & Sufficient \\
\hline $\begin{array}{l}\text { Elli elle ellediğin eller ile elli elle ellenmek ister. } \mathrm{PTT}_{36} \\
(\mathrm{E})\end{array}$ & Normal & Sufficient & Sufficiently & Sufficient \\
\hline $\begin{array}{l}\text { Emel emellerine ulaşmak için erken erken evden kaçıp } \\
\text { evlendi. } \mathrm{PTT}_{43}(\mathrm{E})\end{array}$ & Normal & Sufficient & Sufficiently & Sufficient \\
\hline $\begin{array}{l}\text { Eren evine en erken enikler el ele verdiği zaman } \\
\text { dönebilir. } \mathrm{PTT}_{30}(\mathrm{E})\end{array}$ & Normal & Sufficient & Sufficiently & Sufficient \\
\hline $\begin{array}{l}\text { Esen'in elindeki eski etek, Ersin'in köpeği çaldı kepek. } \\
\mathrm{PTT}_{28}(\mathrm{E})\end{array}$ & Normal & Sufficient & Sufficiently & Sufficient \\
\hline $\begin{array}{l}\text { Eski evlerin içinde var bir elek, elĕ in içinde bir etek, etek } \\
\text { emziğe sarılmuş ezelinden beri orada durur. } \mathrm{PTT}_{38}(\mathrm{E})\end{array}$ & Long & Sufficient & Sufficiently & Sufficient \\
\hline $\begin{array}{l}\text { Eskişehirli edepsiz Edip edebinden edepsizlik edemezdi. } \\
\mathrm{PTT}_{9}(\mathrm{E})\end{array}$ & Normal & Sufficient & Moderate & Average \\
\hline $\begin{array}{l}\text { Eşek eşeğe eş diye eş bulamadan eşsiz eşeğe eş demiş. } \\
\mathrm{PTT}_{7}(\mathrm{E})\end{array}$ & Normal & Sufficient & Moderate & Average \\
\hline Eye büze süse küse süs püs süsletti. PTT $_{5}(\mathrm{E})$ & Short & Medium & Moderate & Sufficient \\
\hline $\begin{array}{l}\text { Gel gidelim gene güne geceye gaglayı giglamadan } \\
\text { gösterelim gangam gardırobun gözünü. } \text { PTT }_{41}(\mathrm{G})\end{array}$ & Long & Sufficient & Sufficiently & Sufficient \\
\hline $\begin{array}{l}\text { Gel Gümüşhane’de gülcü Gülşen'in gününe gidelim } \\
\text { günümüzü gün edelim. } \mathrm{PTT}_{14}(\mathrm{G})\end{array}$ & Normal & Medium & Sufficiently & Sufficient \\
\hline Gele gele göze geldi göremeyecesiceler. $\mathrm{PTT}_{39}(\mathrm{G})$ & Short & Less & Sufficiently & Sufficient \\
\hline $\begin{array}{l}\text { Gelen geleni gele gele giden gideni gide gide güdermiş. } \\
\mathrm{PTT}_{4}(\mathrm{G})\end{array}$ & Normal & Medium & Sufficiently & Sufficient \\
\hline Gelibolu'dan gele gele Gelibolulu gelin geldi. $\mathrm{PTT}_{36}(\mathrm{G})$ & Short & Medium & Sufficiently & Sufficient \\
\hline $\begin{array}{l}\text { Gelin gider gavura, gavur söver karıya, gün görmeyen } \\
\text { gelin girer gavurun kanına. } \mathrm{PTT}_{45}(\mathrm{G})\end{array}$ & Long & Medium & Sufficiently & Sufficient \\
\hline 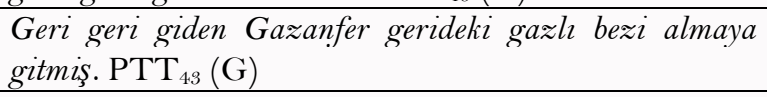 & Normal & Medium & Sufficiently & Sufficient \\
\hline $\begin{array}{l}\text { Gırgırlamayı kes gırıl gırıl gerçekçi gibi gırıl gırıl } \\
\text { gurulda. } \mathrm{PTT}_{42}(\mathrm{G})\end{array}$ & Normal & Medium & Sufficiently & Sufficient \\
\hline Gizli gizli gel git, git gel de götürttürtme. $\mathrm{PTT}_{5}(\mathrm{G})$ & Short & Medium & Sufficiently & Sufficient \\
\hline $\begin{array}{l}\text { Gözlerim gözlerinin gözledĭgi gözleri gözleseydi } \\
\text { gözlerimle gözlerin göz göze gelirdi. } \text { PTT }_{12}(\mathrm{G})\end{array}$ & Normal & Medium & Sufficiently & Sufficient \\
\hline $\begin{array}{l}\text { Gurbet guguk kuşunu gugukturdu, guguktan gurbet } \\
\text { gukturdu. } \mathrm{PTT}_{18}(\mathrm{G})\end{array}$ & Normal & Medium & Sufficiently & Sufficient \\
\hline $\begin{array}{l}\text { Güzel gelinlikli güzel gelin Gana'daki düğ̈̈ne koşup } \\
\text { gelin. } \mathrm{PTT}_{28}(\mathrm{G})\end{array}$ & Normal & Medium & Sufficiently & Sufficient \\
\hline $\begin{array}{l}\dot{\text { It }} \text { iti itti bit biti itti it gitti bit gitti itti bitti gitti. } \mathrm{PTT}_{8} \\
(\mathrm{~T})\end{array}$ & Normal & Sufficient & Sufficiently & Sufficient \\
\hline $\begin{array}{l}\text { Kalın kemikle kalın olmayan kemik karşılaşmış kalın } \\
\text { olanı kalın olmayana "Neden sen de kalın değilsin?" } \\
\text { demiş. } \mathrm{PTT}_{31}(\mathrm{~K})\end{array}$ & Long & Medium & Sufficiently & Sufficient \\
\hline $\begin{array}{l}\text { Kalpsiz katırcı koluyla kaldık da kalpsiz katırc } \\
\text { donakaldı. } \mathrm{PTT}_{35}(\mathrm{~K})\end{array}$ & Normal & Medium & Sufficiently & Sufficient \\
\hline $\begin{array}{l}\text { Kara kedinin kırmızı kurdelesi kıtır kıtır kıttırıldı. } \\
\mathrm{PTT}_{13}(\mathrm{~K})\end{array}$ & Normal & Medium & Sufficiently & Sufficient \\
\hline $\begin{array}{l}\text { Kara Kemal kara kömür ile karalandı, karısını da kara } \\
\text { su ile karaladı. } \mathrm{PTT}_{15}(\mathrm{~K})\end{array}$ & Normal & Medium & Sufficiently & Sufficient \\
\hline $\begin{array}{l}\text { Kartal kalkar dal sarkar, dal sarkar kartal kalkar. } \\
\mathrm{PTT}_{37}(\mathrm{~K})\end{array}$ & Normal & Medium & Sufficiently & Insufficient \\
\hline $\begin{array}{l}\text { Kartal kalkar, dal sarkar, dal sarkar, kartal kalkar. } \\
\mathrm{PTT}_{22}(\mathrm{~K})\end{array}$ & Normal & Medium & Sufficiently & Insufficient \\
\hline 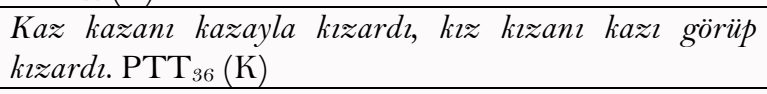 & Normal & Medium & Sufficiently & Sufficient \\
\hline Kekeme kekelemede kelimelere denemeden deneme edemez. & Normal & Sufficient & Sufficiently & Sufficient \\
\hline
\end{tabular}




\begin{tabular}{|c|c|c|c|c|}
\hline $\mathrm{PTT}_{17}(\mathrm{~K})$ & & & & \\
\hline $\begin{array}{l}\text { Kel bakıp tabakta kabak keser, tabak kirllp kelin elini } \\
\text { keser. } \mathrm{PTT}_{46}(\mathrm{~K})\end{array}$ & Normal & Medium & Sufficiently & Sufficient \\
\hline $\begin{array}{l}\text { Kel Kâhya Kâzım, koşarak kurstan kaçarken kuşları } \\
\text { korkutunca kapılmış kuşların korkusuna da hevesi } \\
\text { kursağında kalmış. } \mathrm{PTT}_{14}(\mathrm{~K})\end{array}$ & Long & Sufficient & Sufficiently & Sufficient \\
\hline $\begin{array}{l}\text { Kır kara bir kedi Karayazi'da kara köşede koşuşturuyor. } \\
\operatorname{PTT}_{34}(\mathrm{~K})\end{array}$ & Normal & Medium & Sufficiently & Sufficient \\
\hline Kırk küp kırkının da kulpu kırık küp. $\mathrm{PTT}_{3}(\mathrm{~K})$ & Short & Medium & Sufficiently & Insufficient \\
\hline Kırk küp, kırkının da kulpu kırık, kırık küp. PTT $_{8}(\mathrm{~K})$ & Short & Sufficient & Sufficiently & Insufficient \\
\hline $\begin{array}{l}\text { Kim kem kümleşti kim kem kümleşemeden kemleşti. } \\
\text { PTT }_{33}(\mathrm{~K})\end{array}$ & Normal & Medium & Sufficiently & Sufficient \\
\hline $\begin{array}{l}\text { Kitabın kapağını kapatan kabanlı kadın kitabın } \\
\text { kapağındaki kabanlı kadına kendini kaptırınca kararan } \\
\text { havayı fark edemedi. } \mathrm{PTT}_{7}(\mathrm{~K})\end{array}$ & Long & Sufficient & Sufficiently & Sufficient \\
\hline $\begin{array}{l}\text { Köyde oldu kepek, ketek çaldı köpek, kötek aldı köpek. } \\
\text { PTT }_{26}(\mathrm{~K})\end{array}$ & Normal & Sufficient & Sufficiently & Insufficient \\
\hline $\begin{array}{l}\text { Kurttan kurtulan küçük kurt kurttan kurtuldu da kurt } \\
\text { kendini kürk olup kürksüz kımıldamaktan kurtulamadı. } \\
\text { PTT }_{41}(\mathrm{~K})\end{array}$ & Long & Sufficient & Sufficiently & Sufficient \\
\hline $\begin{array}{l}\text { Okuyan tren garında okumayanlar okudukça okudukları } \\
\text { akıllarına okur demiş. } \operatorname{PTT}_{20}(\mathrm{O})\end{array}$ & Normal & Less & Moderate & Sufficient \\
\hline Oluk oluk olur Olur'un oltasının orkinosu. $\mathrm{PTT}_{36}(\mathrm{O})$ & Short & Medium & Moderate & Sufficient \\
\hline $\begin{array}{l}\text { Onca olan olaydan sonra olanları ona onaylatmam } \\
\text { onadı. } \mathrm{PTT}_{25}(\mathrm{O})\end{array}$ & Normal & Medium & Moderate & Average \\
\hline $\begin{array}{l}\text { Onun oğluna Oya doya doya oya yapmamış mı? } \text { PTT }_{46} \\
(\mathrm{O})\end{array}$ & Normal & Medium & Sufficiently & Sufficient \\
\hline $\begin{array}{l}\text { Oradan oraya otlayan ot obur ortadan otlarken otlamaya } \\
\text { gelen ot oburlar otlatmadan ortadan kayboldu. } \mathrm{PTT}_{7} \\
(\mathrm{O})\end{array}$ & Long & Sufficient & Moderate & Sufficient \\
\hline 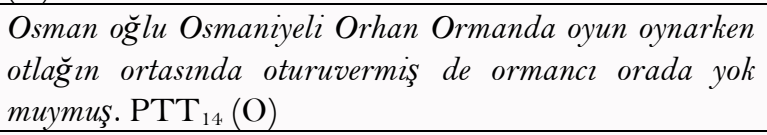 & Long & Sufficient & Moderate & Sufficient \\
\hline $\begin{array}{l}\text { Osman'ın o'suyla, Orhan'ın O'su o gün orada olmuştu. } \\
\text { PTT }_{28}(\mathrm{O})\end{array}$ & Normal & Medium & Sufficiently & Sufficient \\
\hline $\begin{array}{l}\text { Osman'ın oğlu Onur oturup oğlakları otlatmaya koyuldu, } \\
\text { Osman oturan oğlu Onur'u oturup oyalandığını sanıp } \\
\text { oturtmaktan alıkoydu oturtmalıklı Onur'u. } \mathrm{PTT}_{41}(\mathrm{O})\end{array}$ & Long & Sufficient & Moderate & Average \\
\hline $\begin{array}{l}\text { Palavracı Pala Ferit, palasıyla piton parçaladığ } \imath \\
\text { palavrasın herkese duyurdu. } \mathrm{PTT}_{22}(\mathrm{P})\end{array}$ & Normal & Medium & Sufficiently & Sufficient \\
\hline $\begin{array}{l}\text { Paramparça pırasaların pırasa değerleri parıl parıl } \\
\text { parlarken paparazzilerin pırasaları şipşaklamasıdı. } \\
\mathrm{PTT}_{42}(\mathrm{P})\end{array}$ & Long & Sufficient & Moderate & Average \\
\hline $\begin{array}{l}\text { Parayı peşin alan pazarcı parayı bozamadan pırasayı } \\
\text { parça parça paralayıp parayı pul diye paralamış. } \mathrm{PTT}_{7} \\
(\mathrm{P})\end{array}$ & Normal & Sufficient & Sufficiently & Sufficient \\
\hline $\begin{array}{l}\text { Parisli patavatsız Pınar pırasaları parça parça edip } \\
\text { patavatsızlı̆̆ını posterledi. } \mathrm{PTT}_{9}(\mathrm{P})\end{array}$ & Normal & Medium & Moderate & Average \\
\hline $\begin{array}{l}\text { Pasajdan pasakl paspas parasına, parlattı̆̆ } \imath \text { paltosunu } \\
\text { pat küt kaldıran paspasa pasajdan paldır palas bakan } \\
\text { kız. } \mathrm{PTT}_{6}(\mathrm{P})\end{array}$ & Long & Sufficient & Sufficiently & Sufficient \\
\hline $\begin{array}{l}\text { Pasaklı pasajcı verdi pası, pis paspasçı sildi pası. } \text { PTT }_{36} \\
(\mathrm{P})\end{array}$ & Normal & Medium & Sufficiently & Sufficient \\
\hline Patır patır gelen pireler, pitır pitır gittiler. $\mathrm{PTT}_{15}(\mathrm{P})$ & Short & Less & Sufficiently & Sufficient \\
\hline $\begin{array}{l}\text { Pazar Pazar pazara pastırmaya palazlananlar pazar } \\
\text { parasın palazlana palazlana paralanırlar. } \mathrm{PTT}_{4}(\mathrm{P})\end{array}$ & Long & Medium & Sufficiently & Sufficient \\
\hline 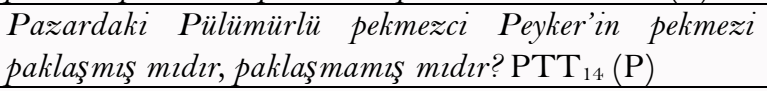 & Normal & Medium & Sufficiently & Sufficient \\
\hline $\begin{array}{l}\text { Peçete paketini parala, paranı peçete paketiyle pakla. } \\
\text { PTT }_{44}(\mathrm{P})\end{array}$ & Normal & Medium & Moderate & Sufficient \\
\hline Pısırık pirasaları patır patır purtlayarak aldı. $\mathrm{PTT}_{5}(\mathrm{P})$ & Short & Less & Moderate & Average \\
\hline $\begin{array}{l}\text { Pir gelip bir gelen Pelin pırasa pırasa saçlarımı partlattı. } \\
\text { PTT }_{43}(\mathrm{P})\end{array}$ & Normal & Medium & Moderate & Average \\
\hline 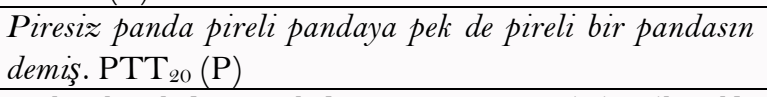 & Normal & Medium & Sufficiently & Sufficient \\
\hline $\begin{array}{l}\text { Tak tek takalayıp tukalayı tüt tut tat tittitretik takla } \\
\text { tuttur. } \mathrm{PTT}_{41}(\mathrm{~T})\end{array}$ & Normal & Sufficient & Sufficiently & Average \\
\hline $\begin{array}{l}\text { Takılarız tezgâha taşı da takı, toka takılmasın } \\
\text { topuğuma. } \mathrm{PTT}_{11}(\mathrm{~T})\end{array}$ & Normal & Medium & Sufficiently & Sufficient \\
\hline $\begin{array}{l}\text { Taksideki Tuzlalı tatlı kız tuzlu tarçınlı tatlı tattı. } \\
\text { PTT }_{28}(\mathrm{~T})\end{array}$ & Short & Medium & Sufficiently & Sufficient \\
\hline $\begin{array}{l}\text { Tamirci Turan Trabzon ve Tekirdağ'da tamı tamına } \\
\text { dört yüz dört tır tamiri yaptı. } \text { PTT }_{31}(\mathrm{~T})\end{array}$ & Normal & Sufficient & Sufficiently & Sufficient \\
\hline Tap tip top kim geliyor trap trip trop. $\mathrm{PTT}_{2}(\mathrm{~T})$ & Short & Medium & Sufficiently & Sufficient \\
\hline
\end{tabular}


Tatlı sudaki tatlı balık tatlıliktan taraland.. $\mathrm{PTT}_{35}(\mathrm{~T})$

Tatliy temiz temiz taslara tabaklara koy da tarlada tahteravallinin üstündeki torbalara taşı. $\mathrm{PTT}_{38}(\mathrm{~T})$

Tedarikli davranan talebeler test kâğtlarını tek tek teslim etti. PTT $_{40}(\mathrm{~T})$

Tepeden tepinerek inen tauşan tepine tepine indiğ tepeden tepinemeden tepeye geri tepindi. $\mathrm{PTT}_{7}(\mathrm{~T})$ Tersinden tepesinden tepilen tilki, tepilmiş ă̆aca tepelenmeden tepenir. $\mathrm{PTT}_{6}(\mathrm{~T})$

Tisır tısır tıslayarak trenle tıkır tıkır geldi. $\mathrm{PTT}_{5}(\mathrm{~T})$

Tokatlıyı tokatlayan tekin ve tek olmayan Tekir dostların topladı. $\mathrm{PTT}_{43}(\mathrm{~T})$

Tükkanda tost tostturulmadl, tost tostturan tukkan taradık aradık. $\mathrm{PTT}_{18}(\mathrm{~T})$

Tümendeki tüfekçi tütüncüyle tütün tüttürdü. $\mathrm{PTT}_{45}(\mathrm{~T})$ Ufuk, ufkunu, umutlarını un ufak edip unut. $\mathrm{PTT}_{24}$ (U)

Ulu Umut usturayla uflaya uflaya uladr. PTT $_{12}$ (U)

Uluyan ulu kurt ile ulumayan ulu kurt el ele ulumuşlar. $\mathrm{PTT}_{31}(\mathrm{U})$

Umarsız umut unuttuğunu unutunca uçak uçtu. PTT $_{19}$ (U)

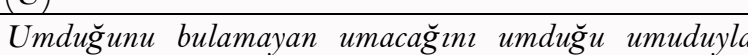
umudunu ummayı ümit ederken umduğunu bulamayıp umulanı umar. $\mathrm{PTT}_{6}(\mathrm{U})$

Umutsuz Umut, ulu orta uğursuz uğursuz uzaklardan bir şeyler umdu. $\mathrm{PTT}_{43}(\mathrm{U})$

Urmiye dereleri uçak bucak uçladı Remzi Paşa uçuğumu purtlattı. $\mathrm{PTT}_{2}(\mathrm{U})$

Uyumak uyumaya uysaydı uykucu Umut uykudan uyandı̆̆ında umutlarını uykudaki Umut'un umuduna bırakmayzp umup uyumaya uyurdu. $\mathrm{PTT}_{4.1}(\mathrm{U})$

Uzak uzak diyarlardan uyarlar bize ulaşırken, ulu uzun boylu budalalar bunlarla uzaktan yakindan uyarlara uymadilar. $\mathrm{PTT}_{38}(\mathrm{U})$

Uzaktan uzăga uluyan kurtlar ufak ufak tufak kufak uluştular. $\mathrm{PTT}_{13}(\mathrm{U})$

Uzaya uzaya giden uzak yol uzadıkça uzayınca uzun adam uzayıp giden uzak yola uzun uzun baktı. $\mathrm{PTT}_{7}$ (U)

Uzun uzayan uzayın uzaklı̆̆ $\imath$ uzun uzamayan uzayın uzaklı̆̆ını uzattı. $\mathrm{PTT}_{33}(\mathrm{U})$

Uzun uzun ulumaları kuru ve duru kuzuları durdurdu. $\mathrm{PTT}_{17}(\mathrm{U})$

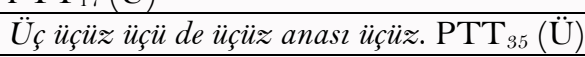

Üf püf üfüleyip tüttürmeli ütüyü, ünü, bünü büsbütün tüttürmeli. PTT $_{40}$ (Ü)

Üflemeden üflemeye başlayan üfürükçü üflediğ $i$ üfürüğe bakıp yine de üflerken üfürttüğ̈̈ üfürü̈ge bir üfürdü. $\mathrm{PTT}_{6}(\ddot{\mathrm{U}})$

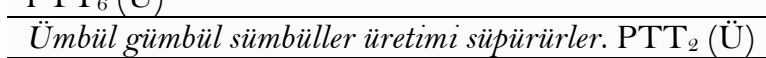

Üstüne üstelik ütücünün ütüyü ütülenmek için ütülettiğ $i$ üfürüldü. $\mathrm{PTT}_{33}$ (Ü)

Üzdü Ümran'ı üzüm, üleştirdi Ümit'e üç üzüm. $\mathrm{PTT}_{36}$ (Ü)

Üzgün üvey üç çocuk üç üzüm için üzülmüş, süzülmüş. $\mathrm{PTT}_{26}(\ddot{\mathrm{U}})$

$\ddot{U}$ zgünce sevgilisi Ümit'i, ümit eden Ümmü ümidini kaybetmedi. $\mathrm{PTT}_{30}(\ddot{\mathrm{U}})$

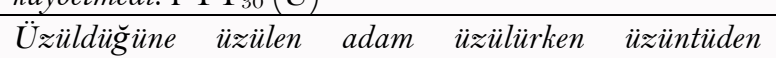
üzüldüğ̈̈nü unutmuş. $\mathrm{PTT}_{7}$ (Ü)

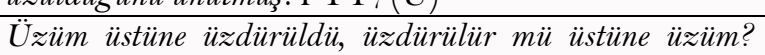
$\operatorname{PTT}_{18}(\ddot{U})$

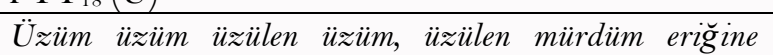
üzülmemesini söyledi. $\mathrm{PTT}_{29}(\ddot{\mathrm{U}})$

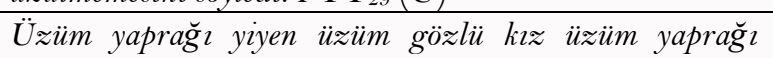
yiyince pek üzülmüş. PTT $_{20}(\ddot{U})$

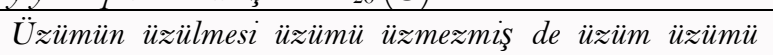
üzermiş. $\mathrm{PTT}_{4}(\ddot{\mathrm{U}})$

Yaban ellerde elle eleğ $i$ eleye dursun, elek de eller de elensin dursun. PTT $_{35}(\mathrm{E})$

re demekle emek derilmez emek yemek demek de emekçiyi emeklilikten etmez. $\mathrm{PTT}_{41}(\mathrm{E})$

\begin{tabular}{|c|c|c|c|}
\hline Short & Medium & Sufficiently & Sufficient \\
\hline Long & Sufficient & Sufficiently & Sufficient \\
\hline Normal & Medium & Moderate & Average \\
\hline Normal & Medium & Sufficiently & Sufficient \\
\hline Normal & Medium & Sufficiently & Sufficient \\
\hline Short & Medium & Sufficiently & Sufficient \\
\hline Normal & Medium & Sufficiently & Sufficient \\
\hline Normal & Sufficient & Moderate & Sufficient \\
\hline Short & Medium & Sufficiently & Sufficient \\
\hline Short & Sufficient & Sufficiently & Sufficient \\
\hline Short & Medium & Sufficiently & Sufficient \\
\hline Normal & Sufficient & Sufficiently & Sufficient \\
\hline Short & Sufficient & Sufficiently & Sufficient \\
\hline Long & Sufficient & Sufficiently & Sufficient \\
\hline Normal & Sufficient & Sufficiently & Sufficient \\
\hline Normal & Medium & Sufficiently & Sufficient \\
\hline Long & Sufficient & Sufficiently & Sufficient \\
\hline Long & Sufficient & Sufficiently & Sufficient \\
\hline Normal & Sufficient & Sufficiently & Sufficient \\
\hline Long & Sufficient & Sufficiently & Sufficient \\
\hline Normal & Sufficient & Sufficiently & Sufficient \\
\hline Normal & Sufficient & Sufficiently & Sufficient \\
\hline Short & Medium & Sufficiently & Sufficient \\
\hline Normal & Sufficient & Sufficiently & Sufficient \\
\hline Long & Sufficient & Sufficiently & Sufficient \\
\hline Short & Medium & Sufficiently & Sufficient \\
\hline Normal & Sufficient & Sufficiently & Sufficient \\
\hline Short & Medium & Sufficiently & Sufficient \\
\hline Normal & Sufficient & Sufficiently & Sufficient \\
\hline Normal & Medium & Sufficiently & Sufficient \\
\hline Normal & Sufficient & Sufficiently & Sufficient \\
\hline Normal & Sufficient & Sufficiently & Sufficient \\
\hline Normal & Sufficient & Sufficiently & Sufficient \\
\hline Normal & Medium & Moderate & Average \\
\hline Normal & Sufficient & Sufficiently & Sufficient \\
\hline Normal & Sufficient & Sufficiently & Sufficient \\
\hline Normal & Sufficient & Sufficiently & Sufficient \\
\hline
\end{tabular}


When Table 6 is examined, it is seen that 149 diction rhymes are suitable for use in diction education studies. In terms of length, 23 of these diction rhymes are short (15.4\%), 98 are normal (65.8\%) and 28 are long (18.8\%). The sound targeted to improve the pronunciation is less (4\%) in 6, medium (49.7\%) in 74 and sufficient $(46.3 \%)$ in 69 of diction rhymes. In terms of entertainment, it was determined that 1 of the diction rhyme is not entertaining. 38 of the diction rhymes have moderate (25.5\%) and 110 of them have enough $(73.8 \%)$ level of entertainment. Regarding the creativity levels of diction rhymes, it is seen that prospective Turkish Teachers are sometimes under the influence of the diction rhymes used before. Of the diction rhymes, 20 have insufficient (13.4\%) and 15 have average (10.1\%) creativity levels. It is seen that $114(76.5 \%)$ diction rhymes have a sufficient level of creativity.

\section{Conclusion and Recommendations}

In this study where how the diction rhyme preparation skills of prospective Turkish teachers is determined, it can be seen that even though the prepared rhymes' sentence lengths are very short in some sentences, they generally have normal sentence lengths. Giving enough place to the target sound desired to be corrected in the rhyme is found to be a positive result.

The fact that rhyme sentences have a fun aspect makes it easier to use them in diction exercises. Thus, they become interesting. This feature has been found to be adequately provided in prepared rhymes. In terms of carrying the characteristics of creativity, it has been observed that rhymes are sometimes written in the same way as they were previously known or put forward with minor changes. Authenticity has been obtained in most of the rhymes though.

Although the prospective Turkish teachers were studying at first grade, they were able to provide the $64.3 \%$ rate of the prepared rhymes in terms of their use in diction exercises. This is an indication that they have sufficient skills in this regard.

It is believed that moving Turkish teachers from their passive listener and the exercise implementer roles to the active creator roles will have positive contributions in speaking education and diction practices in terms of teaching Turkish.

\section{References}

Alyılmaz, C. (2010). Problems of Turkish teaching. Turkish Studies, 5(3), 728-749.Available at: https://doi.org/10.7827/turkishstudies.1629. Aydin, S., \& Akyüz, S. (2017). A brief comparison of the current approaches in teaching pronunciation. Online Submission, 8, 12-15.

Büyükokutan, T. A. (2017). A new environment in the formation and transfer of rhymes: Rhyme sites. Turkish Studies, 12(22), 883-898.

Çifci, M. (2006). Turkish teaching problems. (ed. Gürer Gülsevin and Erdoğan Boz). Contemporary problems of Turkish (pp. 77-135). Ankara: Gazi.

Cox, J. L., Henrichsen, L. E., Tanner, M. W., \& McMurry, B. L. (2019). The needs analysis, design, development, and evaluation of the english pronunciation guide: An esl teachers' guide to pronunciation teaching using online resources. The Electronic Journal for English as a Second Language, 22(4), 1-24.

Dedeoğlu, O. B. (2009). The effect of nursery rhyme on Turkish speaking skills in elementary school 3rd grade students. Unpublished Master's Thesis. İzmir: Dokuz Eylül University Educational Sciences Institute.

Er, O., \& Demir, Ö. (2013). Examination of the opinions of Turkish teacher candidates regarding the usability of speaking skill by Turkish teachers. Turkish Studies, 8(1), 1417-1436.

Erdem, İ. (2013). Speech disorders encountered during speech training and ways to correct them. Adryaman University Journal of Social Sciences Institute Special Issue of Teaching Turkish Education, 6(1 1 ), 415-452.

Erdem, İ. (2013). Speaking education: Theory, practice, measurement and evaluation. Turkish teaching handbook. (ed. Abdurrahman Güzel and Halit Karatay). Ankara: Pegem Academy.

Erdem, M. D., Şengül, M., Gün, M., \& Büyükaslan, A. (2015). Effect of rhyme exercises based on rhyme exercises on speaking skills of Syrian Arabs who learn Turkish as a foreign language. Route Educational and Social Science Journal, 2(2), 1-1 1.

Gündüz, O., \& Şimşek, T. (2014). Narration techniques 2 hands-on speech training manual. Ankara: Graphic Designer.

Hartley, P. (2010). Interpersonal communication. Ankara: Image.

Merriam, S. B. (2013). A guide to qualitative research pattern and practice. (Translation: Selahattin Turan). Ankara: Nobel.

Senyiğit, Y., \& Okur, A. (2019). Speaking skills and pronunciation training in teaching Turkish to foreigners. Mehmet Akif Ersoy University Journal of Education Faculty, 52, 519-549.

Sönmez, V., \& Alacapınar, F. G. (2014). Sampled scientific research methods. Ankara: Anı.

Tanrikulu, F. (2017). Teachers' views on the competence of EBA to meet Turkish lesson learning areas. Journal of Mother Tongue Education, $5(3), 395-416$.

Taşer, S. (2009). Speaking education. Istanbul: Pegasus.

Temizyürek, F. (2007). Improving speaking skill in primary education. Journal of Ankara University Faculty of Educational Sciences, 40(2), 113131.

Temizyürek, F., Erdem, İ., \& Temizkan, M. (2013). Speaking education verbal expression. Ankara: Pegem Academy.

Toker, S., Sevinç, S., \& Önder, Y. V. B. (2018). The effect of rhymes on articulation in vocal education. International Journal of Civilization Studies, 3(1), 321-336.

Türk, O. (2017). A study on Turkish teachers' interest in speech and the language of speech they use in class. Turkish Studies, 12(6), 783-798.

Vural, B. (2007). Accurate and good speech phonetic-diction-articulation with practice and examples. Istanbul: Life.

Yardımcı, M. (2004). Turkish folk poetry from the beginning to the present. Ankara: Product.

Yıldırım, A., \& Şimşek, H. (2013). Qualitative research methods in the social sciences (9th ed.). Ankara: Seçkin. 